\title{
A bound on the number of curves of a given degree through a general point of a projective variety
}

\author{
Jun-Muk Hwang
}

\begin{abstract}
Let $X$ be an irreducible projective variety of dimension $n$ in a projective space and let $x$ be a point of $X$. Denote by $\operatorname{Curves}_{d}(X, x)$ the space of curves of degree $d$ lying on $X$ and passing through $x$. We will show that the number of components of $\operatorname{Curves}_{d}(X, x)$ for any smooth point $x$ outside a subvariety of codimension $\geqslant 2$ is bounded by a number depending only on $n$ and $d$. An effective bound is given. A key ingredient of the proof is an argument from Ein, Küchle and Lazarsfeld's work on Seshadri numbers.
\end{abstract}

\section{Introduction}

This work was motivated by the following result of Landsberg [Lan03].

Theorem 1 [Lan03, Theorem 1]. Let $X$ be an irreducible projective variety of dimension $n$ in a projective space and let $x \in X$ be a general point. Then the number of lines lying on $X$ and passing through $x$ is either infinite or bounded by $n$ !.

It is remarkable that the bound $n$ ! is optimal: it is achieved when $X$ is a smooth hypersurface of degree $n$ in $\mathbb{P}_{n+1}$. However, even if we disregard the optimality of the bound, the uniformity of the bound is already quite remarkable. Namely, the fact that the bound depends only on the dimension $n$ of $X$ is worth noting. When interpreted as such a uniform boundedness result, Theorem 1 naturally leads to the following questions.

Question 1. What about curves of higher degree? Is the number of curves of degree $d>0$ lying on $X$ and passing through a general point $x \in X$ either infinite or bounded by a number depending only on $d$ and $n$ ?

Question 2. What about the case when there are infinitely many lines through a general point $x$ ? Is the number of components of the space of lines lying on $X$ and passing through a general point $x \in X$ bounded by a number depending only on $n$ ?

Question 3. What about non-general points? Is the number of lines lying on $X$ through any given point of $X$ either infinite or bounded by a number depending only on $n$ ?

In Landsberg's proof, the uniformity comes from his earlier result [Lan99] that a line osculating to order $n+1$ at a general point of $X$ must be contained in $X$. The differential geometric argument of [Lan99] using the moving frame method seems difficult to generalize to handle the above questions.

In this paper, we introduce another approach to these questions, using tools from the study of uniform lower bounds for the Seshadri numbers of an ample line bundle at general points of

Received 6 January 2004, accepted in final form 26 May 2004, published online 21 April 2005.

2000 Mathematics Subject Classification 14J40.

Keywords: Chow variety, Seshadri number.

Supported by the Korea Research Foundation Grant (KRF-2002-070-C00003).

This journal is (C) Foundation Compositio Mathematica 2005. 


\section{J.-M. HWANG}

a variety [EKL95, HK03]. Apparently, there is no direct connection between the questions raised above and Seshadri numbers. Nonetheless the arguments used in [EKL95] and [HK03] can be translated to apply here. Using this, we will get effective, albeit non-optimal, answers to Questions 1 and 2. Our result can be stated as follows. Let us denote by $\operatorname{Curves}_{d}(X, x)$ the space of curves of degree $d$ lying on a projective variety $X$ and passing through a point $x \in X$. (See $\S 1.1$ for a precise definition.)

Theorem 2. Let $n$ and $d$ be two positive integers. Let $X$ be an irreducible projective variety of dimension $n$ in a projective space and $x \in X$ be a general point. Then the number of components of $\operatorname{Curves}_{d}(X, x)$ is bounded by

$$
\left(\begin{array}{c}
(2 n+2)\left((2 n d)^{n}-1\right) \\
2 n+1
\end{array}\right)(2 n+2)\left(4 d^{2}-4 d+2\right) .
$$

Regarding Question 3, the answer is plainly no. Let us look at two examples.

Example 1. Let $\mathbb{P}_{1} \times \mathbb{P}_{2} \subset \mathbb{P}_{5}$ be the Segre embedding. Choose a curve $C$ of degree $\ell>0$ in $\mathbb{P}_{2}$. Let $X^{\prime}$ be the surface $\mathbb{P}_{1} \times C \subset \mathbb{P}_{1} \times \mathbb{P}_{2}$. Pick a point $o \in \mathbb{P}_{1}$ and a point $P$ in $\{o\} \times \mathbb{P}_{2} \subset \mathbb{P}_{1} \times \mathbb{P}_{2}$ outside $X^{\prime}$. Let $X \subset \mathbb{P}_{4}$ be the projection of $X^{\prime}$ from $P$. Then the image of $\{o\} \times C$ is a line in $X$ at a general point of which there are at least $\ell+1$ lines. Given any dimension $n \geqslant 2$ and an integer $\ell>0$, by taking the Segre product of $X$ with an irreducible variety of dimension $n-2$ containing no lines, we get an example of an irreducible variety of dimension $n$ where the number of lines through a general point in a hypersurface is finite, but larger than $\ell$.

Example 2. Let $k$ be an odd integer and consider the Fermat surface $X_{0}^{k}+X_{1}^{k}+X_{2}^{k}+X_{3}^{k}=0$ in $\mathbb{P}_{3}$. Then through the point $(1,-1,0,0)$ there are at least $k$ distinct lines defined by $X_{0}+X_{1}=0$ and $X_{2}+e^{2 \pi j \sqrt{-1} / k} X_{3}=0,1 \leqslant j \leqslant k$. Given any dimension $n \geqslant 2$ and an integer $M>0$, by taking the Segre product of the Fermat surface with a smooth variety of dimension $n-2$ containing no lines, we get an example of a smooth variety of dimension $n$ where the number of lines through any point in a codimension-2 subset is finite, but larger than $M$.

These examples suggest that the following result of ours gives a more or less optimal answer to Question 3.

Theorem 3. Let $X$ be an irreducible projective variety of dimension $n$ in a projective space. Then there exists a subvariety $R$ of codimension $\geqslant 2$ in the smooth locus of $X$ such that, for any smooth point of $X$ off $R$, the number of components of $\operatorname{Curves}_{d}(X, x)$ is bounded by a number $\nu_{n, d}$ depending only on $n$ and $d$.

For an explicit value of the bound $\nu_{n, d}$, see Definition 4 .

The rough idea of the proofs of Theorems 2 and 3 is the following. First we will explain in $\S 2$ how to obtain a bound depending on the degree of $X$. Here the main ingredient, in addition to some elementary projective geometry, is the effective bound on the number of components of Chow varieties obtained in recent works on effective bounds on the number of maps dominating varieties of general type (see e.g. [Gue99] and [Tsa98]). Now to prove Theorems 2 and 3, the strategy is to study the foliation on $X$ generated by curves of degree $d$, to be constructed in $\S 3$. This foliation has the property that its general leaf contains all curves of degree $d$ lying on $X$ passing through a general point of the leaf, and it is the foliation of minimal rank with this property. Thus to prove Theorem 2, we may replace $X$ by a leaf of the foliation. The heart of the proof of Theorem 2 is to show that the degree of the leaf can be bounded in terms of $n$ and $d$. This is achieved in $\S 4$ by using an argument from Ein, Küchle and Lazarsfeld's work on Seshadri numbers [EKL95]. The proof of Theorem 3, presented in $\S 5$, is by an induction argument using Theorem 2 and by a study of the foliation in codimension 1 . 


\section{BOUND ON THE NUMBER OF CURVES THROUGH A POINT}

\subsection{Notation and conventions}

(1) Throughout this paper, we will work over the complex numbers.

(2) A variety need not be irreducible, but has finitely many components. For a variety $Y$, $\# Y$ denotes the number of irreducible components of $Y$.

(3) By a curve of degree $d$ on an irreducible projective variety $Y$ in a projective space, we mean an irreducible reduced subvariety of dimension 1 lying on $Y$ which has degree $d$ with respect to the hyperplane line bundle of the projective space.

(4) For an irreducible subvariety $Y$ in a projective space, denote by $\operatorname{Chow}_{1, d}(Y)$ the Chow variety of effective 1-cycles of degree $d$ on $Y$ (see [Kol96, I.3] for the definition). Denote by $\operatorname{Curves}_{d}(Y)$ the quasi-projective subvariety in $\operatorname{Chow}_{1, d}(Y)$ parametrizing curves of degree $d$ on $Y$. For a point $y \in Y$, denote by $\operatorname{Curves}_{d}(Y, y)$ the subvariety of $\operatorname{Curves}_{d}(Y)$ parametrizing members passing through $y$.

(5) We will say that a property holds at a general point of an irreducible quasi-projective variety $Y$ if it holds for a non-empty Zariski-open subset of $Y$. It holds at a very general point if it is satisfied off the union of countably many proper closed subvarieties of $Y$.

\section{A bound depending on the degree of the variety}

Let us start by recalling the following elementary fact.

Proposition 1. Let $X \subset \mathbb{P}_{N}$ be an irreducible projective variety and let $x \in X$ be a smooth point. Let $\pi: \widetilde{X} \rightarrow X$ be the blow-up of $X$ at $x$ and let $E$ be the exceptional divisor. Denote by $H$ the hyperplane divisor on $\mathbb{P}_{N}$. Then $2 \pi^{*} H-E$ is a very ample divisor on $\widetilde{X}$. If the degree of $X$ is $a$, the degree of $\widetilde{X}$ with respect to $2 \pi^{*} H-E$ is $2^{n} a-1$.

Proof. Let $\pi^{\prime}: \widetilde{\mathbb{P}}_{N} \rightarrow \mathbb{P}_{N}$ be the blow-up of $\mathbb{P}_{N}$ at $x$ and let $E^{\prime} \subset \widetilde{\mathbb{P}}_{N}$ be the exceptional divisor. More precisely, $\widetilde{\mathbb{P}}_{N}$ is the subvariety of $\mathbb{P}_{N} \times \mathbb{P}_{N-1}$ which is the closure of the graph of the projection of $\mathbb{P}_{N}$ to a hyperplane $\mathbb{P}_{N-1}$ with the vertex $x$ and $E^{\prime}$ is the inverse image of $x$ in $\widetilde{\mathbb{P}}_{N}$ under the projection $p_{1}: \widetilde{\mathbb{P}}_{N} \rightarrow \mathbb{P}_{N}$. Then $E^{\prime}$ is biregular to $\mathbb{P}_{N-1}$ by the projection $p_{2}: \widetilde{\mathbb{P}}_{N} \rightarrow \mathbb{P}_{N-1}$. Let $H$ be the hyperplane divisor on $\mathbb{P}_{N}$ and let $H^{\prime}$ be the hyperplane divisor on $\mathbb{P}_{N-1}$. Then the divisor

$$
\left.\left(p_{1}^{*} H+p_{2}^{*} H^{\prime}\right)\right|_{\widetilde{\mathbb{P}}_{N}}=2 \pi^{*} H-E^{\prime}
$$

is very ample on $\widetilde{\mathbb{P}}_{N}$. The proper image of $X$ in $\widetilde{\mathbb{P}}_{N}$ is $\widetilde{X}$ and the divisor $E \subset \widetilde{X}$ is just the restriction of $E^{\prime}$ to $\widetilde{X}$. Thus $2 \pi^{*} H-E=\left.\left(2 \pi^{* *} H-E^{\prime}\right)\right|_{\widetilde{X}}$ is very ample on $\widetilde{X}$. The degree is

$$
\left(2 \pi^{*} H-E\right)^{n}=2^{n} a-1
$$

from $a=H^{n}$ and $E^{n}=(-1)^{n-1}$.

Proposition 2. Let $x$ be a smooth point of an irreducible projective variety $X \subset \mathbb{P}_{N}$. Assume that a general member of each component of $\operatorname{Curves}_{d}(X, x)$ has multiplicity $\leqslant m$ at $x$ for some positive integer $m$. Let $\pi: \widetilde{X} \rightarrow X$ be the blow-up of $X$ at $x$. Identify $\widetilde{X}$ as a subvariety of a projective space via the very ample divisor $2 \pi^{*} H-E$ of Proposition 1. Then

$$
\text { \#Curves }_{d}(X, x) \leqslant \sum_{i=1}^{m} \# \operatorname{Curves}_{2 d-i}(\tilde{X}) \text {. }
$$

Proof. Fix an integer $i, 1 \leqslant i \leqslant m$. Let $\operatorname{Curves}_{2 d-i}^{\prime}(\tilde{X})$ be the union of the components of $\operatorname{Curves}_{2 d-i}(\widetilde{X})$ whose members have intersection number $i$ with $E$. Any member of $\operatorname{Curves}_{2 d-i}^{\prime}(\widetilde{X})$ is sent by $\pi$ to a curve of degree $d$ passing through $x$ which has multiplicity $i$ at $x$. This induces 


\section{J.-M. HWANG}

a morphism

$$
\pi_{*}: \bigcup_{i=1}^{m} \operatorname{Curves}_{2 d-i}^{\prime}(\tilde{X}) \rightarrow \operatorname{Curves}_{d}(X, x) .
$$

Since a general member of each irreducible component of $\operatorname{Curves}_{d}(X, x)$ has multiplicity $\leqslant m$, the morphism $\pi_{*}$ is dominant on each irreducible component of $\operatorname{Curves}_{d}(X, x)$. Thus

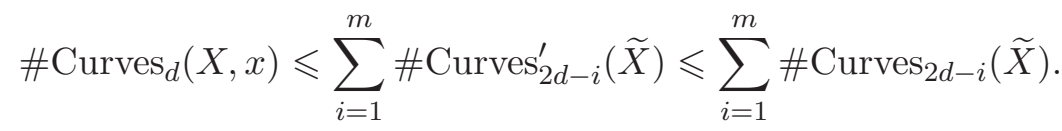

Proposition 3. Let $X \subset \mathbb{P}_{N}$ be an irreducible projective variety and let $x$ be a general point of $X$. Then a general member of any component of $\operatorname{Curves}_{d}(X, x)$ is smooth at $x$.

Proof. Let $B$ be a component of $\operatorname{Curves}_{d}(X)$ whose members sweep out an open subset of $X$ and let $G \subset B \times X$ be the subvariety defined by the incidence relation

$$
G=\left\{(b, x) \in B \times X, x \in C_{b}\right\},
$$

where $C_{b}$ denotes the curve in $X$ corresponding to $b \in B$. It is clear that $G$ contains a unique irreducible component $G^{o}$ of dimension $\operatorname{dim} B+1$. There exists an open subset $X^{\prime} \subset X$ such that, for each $x \in X^{\prime}$, the intersection $\operatorname{pr}_{X}^{-1}(x) \cap G$ is contained in $G^{o}$. By shrinking $X^{\prime}$, we may assume that each component of $\operatorname{pr}_{X}^{-1}(x) \cap G$ has dimension $\operatorname{dim} B+1-\operatorname{dim} X$ for each $x \in X^{\prime}$. Let $S_{x} \subset G \cap p r_{X}^{-1}(x)$ be the subvariety defined by

$$
S_{x}:=\left\{(b, x), C_{b} \text { is singular at } x\right\} .
$$

Suppose $S_{x}$ contains some components of $G \cap p r_{X}^{-1}(x)$ for general $x \in X^{\prime}$. Then the union of the $S_{x}$ as $x$ varies defines a subset of $G$ of dimension $\operatorname{dim} B+1$. This implies that, for a general $x \in X^{\prime}$, we have the equality $S_{x}=G \cap \operatorname{pr}_{X}^{-1}(x)$. This is absurd because some member of $B$ must be smooth at $x$. Hence $S_{x}$ contains no component of $G \cap p r_{X}^{-1}(x)$ for a general $x \in X$. Since this is true for any finitely many possible choices of the component $B$, Proposition 3 is proved.

We will use an effective bound on the number of components of the Chow variety. There are a number of results obtained in this direction (see e.g. [Gue99] and [Tsa98]). For example, the bound given in [Gue99] implies the following proposition.

Proposition 4 [Hei03, Proposition 3.6]. The number of components of the Chow variety Chow $_{k, \delta}(Y)$ parametrizing subvarieties of dimension $k$ and degree $\delta$ in a projective variety $Y$ of degree $\delta^{\prime}$ in $\mathbb{P}_{N^{\prime}}$ is bounded by

$$
\left(\begin{array}{c}
\left(N^{\prime}+1\right) \max \left\{\delta, \delta^{\prime}\right\} \\
N^{\prime}
\end{array}\right)\left(\begin{array}{c}
\left.N^{\prime}+1\right)\left[\delta\left(\begin{array}{c}
\delta+k-1 \\
k
\end{array}\right)+\left(\begin{array}{c}
\delta+k-1 \\
k-1
\end{array}\right)\right] \\
.
\end{array}\right.
$$

Definition 1. Given positive integers $n, d$ and $a$, define

$$
\mu_{n, d, a}:=\sum_{i=1}^{d}\left(\begin{array}{c}
(2 n+2) \max \left\{2 d-i, 2^{n} a-1\right\} \\
2 n+1
\end{array}\right)^{(2 n+2)\left(4 d^{2}-4 d i+i^{2}+1\right)}
$$

and

$$
\lambda_{n, d, a}:=\left(\begin{array}{c}
(2 n+2) \max \left\{2 d-1,2^{n} a-1\right\} \\
2 n+1
\end{array}\right)^{(2 n+2)\left(4 d^{2}-4 d+2\right)} .
$$

Proposition 5. Let $X \subset \mathbb{P}_{N}$ be an irreducible projective variety of dimension $n$ and degree $a$. Let $x$ be a smooth point of $X$. Then

$$
\# \operatorname{Curves}_{d}(X, x) \leqslant \mu_{n, d, a} .
$$


If furthermore $x$ is a general point of $X$, then

$$
\# \operatorname{Curves}_{d}(X, x) \leqslant \lambda_{n, d, a} .
$$

Proof. Using Proposition 2, we will bound $\# \operatorname{Curves}_{2 d-i}(\widetilde{X})$ for $1 \leqslant i \leqslant m$. We may project $\tilde{X}$ to $\mathbb{P}_{2 n+1}$ to count \#Curves $2 d-i(\widetilde{X})$. Thus we can use Proposition 4 with $N^{\prime}=2 n+1, \delta=2 d-i$, $\delta^{\prime}=2^{n} a-1$ and $k=1$. Noting that the multiplicity at $x$ of any member of $\operatorname{Curves}_{d}(X, x)$ is bounded by $m=d$, we get the first inequality. For a general point $x \in X$, we can set $m=1$ in Proposition 2 by Proposition 3, which gives the second inequality.

\section{Foliation generated by curves of degree $d$}

Let $X$ be an irreducible projective variety of dimension $n$ in a projective space. Fix a positive integer $d$. The goal of this section is to construct a foliation of minimal rank on an open subset of $X$ such that members of $\operatorname{Curves}_{d}(X, x)$ lie in the closure of the leaf through $x$ for a general $x \in X$. The construction is similar to the construction of Seshadri-exceptional foliation in [HK03]. Here we will do it with some more care because we have to study the foliation in codimension 1 for Theorem 3. Let us start with the definition of a foliation. This may not be the standard definition, but it will be convenient for us.

Definition 2. Let $X$ be an irreducible projective variety. Denote by $\operatorname{Sm}(X)$ the smooth locus of $X$. A subsheaf $\mathcal{F}$ of the tangent sheaf $T_{S m(X)}$ of $S m(X)$ is called a foliation on $X$ if it satisfies the following two conditions.

(1) The quotient $T_{S m(X)} / \mathcal{F}$ is torsion-free on $\operatorname{Sm}(X)$. This implies that the open subset

$$
\operatorname{Dom}(\mathcal{F}):=\left\{x \in \operatorname{Sm}(X), T_{\operatorname{Sm}(X)} / \mathcal{F} \text { is locally free at } x\right\}
$$

is the complement of a subvariety of codimension $\geqslant 2$ in $\operatorname{Sm}(X)$.

(2) For each $x \in \operatorname{Dom}(\mathcal{F})$, there exists a complex analytic submanifold $\mathcal{F}_{x} \subset \operatorname{Dom}(\mathcal{F})$ passing through $x$, called the $x$-leaf of $\mathcal{F}$, such that the fiber of $\mathcal{F}$ at each point $y$ of $\mathcal{F}_{x}$ is the tangent space of $\mathcal{F}_{x}$ at $y$.

Proposition 6. Let $X$ be an irreducible projective variety. Suppose that, for each very general point $x \in X$, we can assign an irreducible projective subvariety $Z_{x}$ such that, for a very general point $x \in X$ and a very general point $y \in Z_{x}$, the two subvarieties $Z_{x}$ and $Z_{y}$ coincide. Then there exists a unique foliation $\mathcal{F}$ on $X$ such that the $x$-leaf $\mathcal{F}_{x}$ is an open subset of $Z_{x}$ for a very general $x$.

Proof. By the countability of the components of the Hilbert scheme of $X$, there exists an irreducible family of subvarieties $\rho: \mathcal{U} \rightarrow \mathcal{D}$ for some subscheme $\mathcal{D} \subset \operatorname{Hilb}(X)$ such that, for a very general point $x \in X$, the subvariety $Z_{x}$ is the image of a fiber of $\rho$ by the evaluation morphism $\eta: \mathcal{U} \rightarrow X$. The assumption that $Z_{x}=Z_{y}$ for a very general $x \in X$ and a very general $y \in Z_{x}$ implies that $\eta$ is birational. Consider the subsheaf $\mathcal{F}^{\prime}$ of the tangent sheaf of $\operatorname{Sm}(X)$ defined by the push-forward of the relative tangent sheaf of $\rho$ restricted to $\eta^{-1}(S m(X))$. Define $\mathcal{F}$ to be the double-dual of $\mathcal{F}^{\prime}$. It is obvious that $\mathcal{F}$ is the unique foliation satisfying the desired property.

Proposition 7. Let $X$ be an irreducible projective variety of dimension $n$ in a projective space. Fix a positive integer $d$. Then there exists a unique foliation $\mathcal{F}$ on $X$, which we will call the foliation generated by curves of degree $d$, with the following properties.

(i) Each leaf of the foliation is a smooth quasi-projective subvariety in $\operatorname{Dom}(\mathcal{F})$.

(ii) For a general point $x \in X$, all members of $\operatorname{Curves}_{d}(X, x)$ are contained in the closure of the leaf of the foliation passing through $x$. 


\section{J.-M. HWANG}

(iii) The foliation $\mathcal{F}$ is minimal with respect to (ii). In other words, if there exists a foliation $\mathcal{G}$ satisfying (ii) then $\mathcal{F}_{x} \subset \mathcal{G}_{x}$ for a general point $x \in X$.

Proof. For each $x \in X$, define

$$
C_{x}:=\text { closure of } \bigcup_{[C] \in \operatorname{Curves}_{d}(X, x)} C .
$$

Then $C_{x}$ is a projective subvariety in $X$, not necessarily of pure dimension. Choose a smooth affine open subset $T \subset X$ and consider the incidence relation $\mathcal{C} \subset T \times X$ defined by

$$
\mathcal{C}=\left\{(t, x), x \in C_{t}\right\} .
$$

We may assume that the projection $p r_{T}: \mathcal{C} \rightarrow T$ is flat by replacing $T$ by an open subset of $T$. Let us make this assumption. For an irreducible subvariety $W \subset X$ intersecting $T$, let

$$
C_{W}:=\text { closure of } \bigcup_{s \in W \cap T} C_{s} .
$$

This subvariety $C_{W}$ of $X$ is not necessarily irreducible. But each component of $C_{W}$ contains $W$ by the flatness of $\mathrm{pr}_{T}: \mathcal{C} \rightarrow T$. This implies that either every component of $C_{W}$ has dimension strictly larger than $W$ or $C_{W}=W$. Note that when $W$ is one point $x \in T, C_{W}=C_{x}$.

An irreducible subvariety $W$ is said to be saturated if $C_{W}=W$. For each $x \in T$, there exists a unique minimal saturated subvariety $Z_{x}$ containing $x$ constructed as follows. Let $Z_{x}^{1}$ be a component of $C_{x}$ and inductively define $Z_{x}^{i+1}$ to be a component of $C_{Z_{x}^{i}}$. Then $\operatorname{dim}\left(Z_{x}^{i+1}\right)>\operatorname{dim}\left(Z_{x}^{i}\right)$ or $Z_{x}^{i}=Z_{x}^{i+1}=Z_{x}^{i+2}=\cdots$. Thus $Z_{x}^{n}=Z_{x}^{n+1}=\cdots$. Define $Z_{x}=Z_{x}^{n}$. Then $Z_{x}$ is saturated. We claim that any saturated subvariety containing $x$ contains $Z_{x}$. In fact, if $W$ is a saturated subvariety and $W^{\prime} \subset W$ is any irreducible subvariety of $W$ intersecting $T$, then $C_{W^{\prime}} \subset W$. Thus if $x \in W$, then $Z_{x}^{i} \subset W$ inductively for all $i$.

We claim that $Z_{x}=Z_{y}$ for a very general $x \in X$ and a very general $y \in Z_{x}$. Assuming the claim, let us finish the proof of Proposition 7. By Proposition 6, we get a foliation $\mathcal{F}$ on $X$ such that the $x$-leaf $\mathcal{F}_{x}$ is an open subset of $Z_{x}$ for a very general $x$. This implies that, for a very general $x$, the $x$-leaf $\mathcal{F}_{x}$ is quasi-projective and contains all members of $\operatorname{Curves}_{d}(X, x)$. But then the same holds for a general $x$. This shows properties (i) and (ii). The condition (iii) follows from the fact that $Z_{x}$ is the minimal saturated subvariety containing $x$ for a general $x$.

Now to prove the claim, notice that $\operatorname{dim} Z_{x}=\operatorname{dim} Z_{y}$ for very general $x$ and $y$. For a very general $x \in X$ and a very general $y \in Z_{x}$, we have $Z_{y} \subset Z_{x}$ by the saturatedness of $Z_{x}$. Thus $Z_{x}=Z_{y}$ by $\operatorname{dim} Z_{x}=\operatorname{dim} Z_{y}$.

\section{Proof of Theorem 2}

Definition 3. Given positive integers $n$ and $d$, define

$$
\lambda_{n, d}:=\lambda_{n, d,(n d)^{n}}=\left(\begin{array}{c}
(2 n+2)\left((2 n d)^{n}-1\right) \\
2 n+1
\end{array}\right)^{(2 n+2)\left(4 d^{2}-4 d+2\right)} .
$$

For a fixed $d, \lambda_{n, d}$ is an increasing function of $n$.

We can restate Theorem 2 as follows.

Theorem 2 (restated). Let $n$ and $d$ be two positive integers. Let $X$ be an irreducible projective variety of dimension $n$ in a projective space and $x \in X$ be a general point. Then

$$
\text { \# } \operatorname{Curves}_{d}(X, x) \leqslant \lambda_{n, d} \text {. }
$$

The heart of the proof of Theorem 2 is the following proposition. 
Proposition 8. Let $X$ be a projective variety of dimension $n$ in $\mathbb{P}_{N}$. Fix a positive integer $d$. Assume that the foliation $\mathcal{F}$ generated by curves of degree $d$ has rank $n$. Then the degree of $X$ is bounded by $(n d)^{n}$.

Proof. The proof is a translation of [HK03, proof of Theorem 1]. We start by recalling the definition of the multiplicity of an effective divisor along an irreducible subvariety. For an effective divisor $D$ on a variety $Y$ and a non-singular point $y \in Y$, let $m_{y}(D)$ denote the multiplicity of $D$ at $y$. For an irreducible subvariety $Z \subset Y$ intersecting the smooth locus of $Y$, let $m_{Z}(D)$ denote $m_{y}(D)$ at a general point $y \in Z$.

Now assume that the degree of $X$ is strictly bigger than $(n d)^{n}$. Using the notation in the proof of Proposition 7, let $\Gamma \subset X \times T$ be the closure of the graph of the inclusion $T \subset X$. From the relation between the degree and the Hilbert polynomial of $X$,

$$
\operatorname{dim} H^{0}(X, \mathcal{O}(k H)) \geqslant \frac{(n d)^{n}}{n !} k^{n}+O\left(k^{n-1}\right) .
$$

As in [EKL95, 3.8], this implies that, if $k \gg 0$, there exists a divisor $D \in\left|\mathcal{O}_{X \times T}\left(p r_{1}^{*}(k H)\right)\right|$ with $m_{\Gamma}(E)>k d n$.

The following lemma is essentially equal to [EKL95, Lemma 3.5.1] or [HK03, Lemma 1]. Its proof will be omitted.

Lemma 1. Let $Z \subset X \times T$ be an irreducible closed subvariety dominating both $X$ and $T$. Then there exists an irreducible closed subvariety $C Z \subset X \times T$ having the following properties:

(i) $Z \subset C Z$;

(ii) for general $t \in T$ with the fiber $Z_{t}$ intersecting $T$, the fiber $(C Z)_{t} \subset X$ consists of some components of $C_{Z_{t}}$. Here $Z_{t}$ is regarded as a subvariety of $X$ by the projection $X \times T \rightarrow X$ and $C_{Z_{t}}$ denotes the subvariety constructed in the proof of Proposition 7.

Using Lemma 1 as in [EKL95, 3.7], we construct a nested sequence of irreducible subvarieties

$$
Z_{0} \subset Z_{1} \subset \cdots \subset Z_{i} \subset \cdots \subset Z_{n}
$$

in $X \times T$ as follows. Set $Z_{0}=\Gamma$. Then inductively define $Z_{i+1}=C Z_{i}$. From the construction, $\left(Z_{n}\right)_{t}=Z_{t}$ for a general $t \in T$ and $Z_{n+1}=Z_{n}$. By the assumption that the rank of $\mathcal{F}$ is $n$, we have $X=Z_{x}$ for a very general $x$. This implies that $Z_{n}=T \times X$.

Consider the multiplicities $m_{Z_{i}}(D)$. We have

$$
m_{Z_{0}}(D)=m_{\Gamma}(D)>k d n, \quad m_{Z_{n}}(D)=m_{X \times T}(D)=0 .
$$

It follows that there is at least one index $i, 0 \leqslant i \leqslant n-1$, such that

$$
m_{Z_{i}}(D)-m_{Z_{i+1}}(D)>k d \text {. }
$$

Now we use the following result from [EKL95].

Lemma 2 [EKL95, Proposition 2.3]. Let $Y$ and $T$ be smooth irreducible varieties, with $T$ affine, and suppose that $Z \subset V \subset Y \times T$ are irreducible subvarieties such that $V$ dominates $Y$. Let $L$ be a line bundle on $Y$, and suppose given on $Y \times T$ a divisor $D \in\left|p r_{Y}^{*} L\right|$. Then there exists a divisor $D^{\prime} \in\left|p_{Y}^{*} L\right|$ on $Y \times T$ whose support does not contain $V$ such that

$$
m_{Z}\left(D^{\prime}\right) \geqslant m_{Z}(D)-m_{V}(D) .
$$

First, let us assume that $X$ is smooth. By Lemma 2, applied to $L=H$ and $Y=X$, there exists a divisor $D^{\prime} \in\left|\mathcal{O}_{X \times T}\left(p r_{X}^{*}(k H)\right)\right|$ such that $m_{Z_{i}}\left(D^{\prime}\right)>k d$ and $Z_{i+1}$ is not contained in the support of $D^{\prime}$. Then for a general $t \in T$ and $x \in\left(Z_{i}\right)_{t}, m_{x}\left(D_{t}^{\prime}\right)>k d$ as in [EKL95, (3.9)]. 


\section{J.-M. HWANG}

But there exists a curve $C^{\prime}$ of degree $d$ on $X$ passing through $x$ which is contained in $\left(Z_{i+1}\right)_{t}$, but not contained in the support of $D_{t}^{\prime}$. Thus we get the contradiction

$$
k d=k\left(H \cdot C^{\prime}\right)=D_{t}^{\prime} \cdot C^{\prime} \geqslant m_{x}\left(D_{t}^{\prime}\right)>k d .
$$

Now when $X$ has singularity, apply the same argument to $Y=\widehat{X}$, a desingularization of $X$. We have

$$
m_{\widehat{Z}_{i}}(\widehat{D})-m_{\widehat{Z}_{i+1}}(\widehat{D})>k d
$$

for the proper images $\widehat{Z}_{i}$ and $\widehat{Z}_{i+1}$ in $\widehat{X}$ of $Z_{i}$ and $Z_{i+1}$ and the pull-back divisor $\widehat{D}$. Thus the same contradiction occurs. This completes the proof of Proposition 8.

Proof of Theorem 2. We will use induction on the dimension $n$. It is obvious for $n=1$. Suppose the rank of $\mathcal{F}$ is $n$. Then the bound follows from Proposition 5 with $a=(n d)^{n}$ by Proposition 8. Suppose the rank of $\mathcal{F}$ is $r<n$. If $r=0$, or, equivalently, $\operatorname{Curves}_{d}(X, x)$ is empty for a general $x$, there is nothing to prove. So let us assume that $r \geqslant 1$. For a general $x \in X$, we may assume that $x$ is a general point of $\mathcal{F}_{y}$ for some $y \in X$. By Proposition 7 , part (ii) we have

$$
\# \operatorname{Curves}_{d}(X, x)=\# \operatorname{Curves}_{d}\left(\mathcal{F}_{y}, x\right) .
$$

But

$$
\text { \# } \operatorname{Curves}_{d}\left(\mathcal{F}_{y}, x\right) \leqslant \lambda_{r, d} \leqslant \lambda_{n, d}
$$

by the induction hypothesis. This proves Theorem 2 .

\section{Proof of Theorem 3}

Definition 4. Given positive integers $n$ and $d$, define inductively

$$
\begin{aligned}
\nu_{1, d} & :=1, \\
\nu_{n, d} & :=\max \left\{\lambda_{n-1, d}+\nu_{n-1, d}, \quad \mu_{n, d,(n d)^{n}} .\right.
\end{aligned}
$$

For a fixed $d$, it is an increasing function of $n$.

We can restate Theorem 3 as follows.

TheOREm 3 (restated). Let $X$ be an irreducible projective variety of dimension $n$ in a projective space. Let $R$ be the subvariety defined by

$$
R:=\left\{x \in \operatorname{Sm}(X), \# \operatorname{Curves}_{d}(X, x)>\nu_{n, d}\right\} .
$$

Then the codimension of $R \subset S m(X)$ is $\geqslant 2$.

Proof. We will use induction on the dimension $n$. Theorem 3 is obvious for $n=1$.

Let $\mathcal{F}$ be the foliation generated by the curves of degree $d$. If the rank of $\mathcal{F}$ is $n$, the degree of $X$ is bounded by $(n d)^{n}$. Thus by Proposition 5 , we get

$$
\# \operatorname{Curves}_{d}(X, x) \leqslant \mu_{n, d,(n d)^{n}}
$$

at any smooth point $x \in X$. In other words, $R=\emptyset$.

Now suppose the rank of $\mathcal{F}$ is $r<m$ and $R$ contains a component $V$ of codimension 1 . Let $v$ be a general point of $V$. Then $v \in \operatorname{Dom}(\mathcal{F})$ from Definition 2, condition (1). Let $F$ be the closure of the $v$-leaf $\mathcal{F}_{v}$. When $r>0$, the set $F \cap V$ is of codimension $\leqslant 1$ in $F$. By slightly moving $v$, we may assume that $v$ is a smooth point of $F$ outside any given subset of codimension $\geqslant 2$ in $F$. Then, by the induction hypothesis, we have

$$
\text { \# } \operatorname{Curves}_{d}(F, v) \leqslant \nu_{r, d} \text {. }
$$

The same inequality holds for the case $r=0$ because $\# \operatorname{Curves}_{d}(F, v)=0$ in that case. 
Let $\operatorname{Curves}_{d}^{\mathrm{s}}(X)$ be the union of components of $\operatorname{Curves}_{d}(X)$ whose members sweep out an open subset of $X$, the superscript s indicating 'sweeping'. Let $\operatorname{Curves}_{d}^{\mathrm{ns}}(X)$ be the union of the other components of $\operatorname{Curves}_{d}(X)$, the superscript ns indicating 'non-sweeping'. For a point $x \in X$, define

$$
\begin{aligned}
\operatorname{Curves}_{d}^{\mathrm{s}}(X, x) & :=\operatorname{Curves}_{d}(X, x) \cap \operatorname{Curves}_{d}^{\mathrm{s}}(X), \\
\operatorname{Curves}_{d}^{\mathrm{ns}}(X, x) & :=\operatorname{Curves}_{d}(X, x) \cap \operatorname{Curves}_{d}^{\mathrm{ns}}(X) .
\end{aligned}
$$

By definition,

$$
\operatorname{Curves}_{d}(X, x)=\operatorname{Curves}_{d}^{\mathrm{s}}(X, x) \cup \operatorname{Curves}_{d}^{\mathrm{ns}}(X, x),
$$

which is not necessarily a disjoint union.

Lemma 3. The subvariety

$$
\left\{x \in X, \# \operatorname{Curves}_{d}^{\mathrm{ns}}(X, x)>\lambda_{n-1, d}\right\}
$$

has codimension $\geqslant 2$ in $X$.

Proof. Let Loc ${ }^{\mathrm{ns}}$ be the subvariety of dimension $\leqslant n-1$ in $X$ which is the closure of the union of members of Curves ${ }_{d}^{\mathrm{ns}}(X)$. If $y \in X-\operatorname{Loc}^{\mathrm{ns}}$, then

$$
\operatorname{Curves}_{d}^{\mathrm{ns}}(X, y)=\emptyset
$$

At a general point $y$ of a component $Y$ of $\operatorname{Loc}^{\text {ns }}$,

$$
\text { \# } \operatorname{Curves}_{d}(Y, y) \leqslant \lambda_{n-1, d}
$$

by Theorem 2. Since there is no other component of $\operatorname{Loc}^{\text {ns }}$ containing $y$ by the generality of $y$,

$$
\text { \#Curves } \mathrm{ns}_{d}(X, y)=\# \operatorname{Curves}_{d}(Y, y) \leqslant \lambda_{n-1, d} \text {. }
$$

This proves the lemma.

Lemma 4. Suppose $x$ is a point on $\operatorname{Dom}(\mathcal{F})$. Then any member of $\operatorname{Curves}_{d}^{\mathrm{s}}(X, x)$ is contained in the closure $F$ of the $x$-leaf $\mathcal{F}_{x}$; in other words,

$$
\operatorname{Curves}_{d}^{\mathrm{s}}(X, x) \subset \operatorname{Curves}_{d}(F, x) .
$$

In particular, $\# \operatorname{Curves}_{d}^{\mathrm{s}}(X, x) \leqslant \# \operatorname{Curves}_{d}(F, x)$ if $x \in \operatorname{Dom}(\mathcal{F})$.

Proof. Suppose not. Let $C$ be a member of $\operatorname{Curves}_{d}^{\mathrm{s}}(X, x)$ which is not contained in $F$. Deformations of $C$ sweep out an open subset of $X$ by the definition of $\operatorname{Curves}_{d}^{\mathrm{s}}(X, x)$. Since $C$ is not contained in $F$, a general deformation of $C$ will not be tangent to the foliation $\mathcal{F}$. Thus at a general point $y \in X$, there is a curve of degree $d$ through $y$ which is not contained in the closure of the leaf of $\mathcal{F}$ through $y$. This is a contradiction to Proposition 7, part (ii), and proves the lemma.

From Lemmas 3 and 4 , if $v$ is a general point of $V \cap \operatorname{Dom}(\mathcal{F})$, then

$$
\begin{aligned}
\text { \#Curves }_{d}(X, v) & \leqslant \# \operatorname{Curves}_{d}^{\mathrm{ns}}(X, v)+\# \operatorname{Curves}_{d}^{\mathrm{s}}(X, v) \\
& =\# \operatorname{Curves}_{d}^{\mathrm{ns}}(X, v)+\# \operatorname{Curves}_{d}(F, v) \\
& \leqslant \lambda_{n-1, d}+\nu_{r, d} \\
& \leqslant \lambda_{n-1, d}+\nu_{n-1, d} \\
& \leqslant \nu_{n, d} .
\end{aligned}
$$

This contradicts the choice of $v \in R$. This completes the proof of Theorem 3 . 


\section{BOUND ON THE NUMBER OF CURVES THROUGH A POINT}

\section{ACKNOWLEDGEMENTS}

This work was done while the author was visiting the Mathematics Department of Harvard University for the year 2003-2004. He is grateful for the hospitality and the financial support provided by Professor Yum-Tong Siu and Harvard University. He would like to thank Dr. Gordon Heier for showing him the preprint [Hei03] and the references [Gue99, Tsa98].

\section{REFERENCES}

EKL95 L. Ein, O. Küchle and R. Lazarsfeld, Local positivity of ample line bundles, J. Differential Geom. 42 (1995), 193-219.

Gue99 L. Guerra, Complexity of Chow varieties and number of morphisms on surfaces of general type, Manuscripta Math. 98 (1999), 1-8.

Hei03 G. Heier, Effective finiteness theorems for maps between canonically polarized compact complex manifolds, Preprint (2003), alg-geom/0311086.

HK03 J.-M. Hwang and J. Keum, Seshadri-exceptional foliations, Math. Ann. 325 (2003), 287-297.

Kol96 J. Kollár, Rational curves on algebraic varieties, Ergeb. Math. Grenzgeb. (3), vol. 32 (Springer, Berlin, 1996).

Lan99 J. M. Landsberg, Is a linear space contained in a submanifold? On the number of derivatives needed to tell, J. reine angew. Math. 508 (1999), 53-60.

Lan03 J. M. Landsberg, Lines on projective varieties, J. reine angew. Math. 562 (2003), 1-3.

Tsa98 I.-H. Tsai, Chow varieties and finiteness theorems for dominant maps, J. Algebraic Geom. 7 (1998), $611-625$.

Jun-Muk Hwang jmhwang@kias.re.kr

Korea Institute for Advanced Study, 207-43 Cheongryangri-dong, Seoul, 130-722, Korea 